\title{
Thermodynamics of QCD low-energy models and the derivative expansion of the effective action
}

\author{
Jens Braun ${ }^{1,2}$ \\ ${ }^{1}$ Theoretisch-Physikalisches Institut, Friedrich-Schiller-Universität Jena, Max-Wien-Platz 1, D-07r43 Jena, Germany \\ ${ }^{2}$ TRIUMF, 4004 Wesbrook Mall, Vancouver, BC V6T 2A3, Canada
}

(Dated: October 22, 2018)

\begin{abstract}
We study the equation of state of a strongly interacting theory of relativistic bosons and chiral fermions in the vicinity and above the chiral phase transition temperature. Our model resembles presently used low-energy models of QCD in many ways, but its simplicity allows us to study systematically various approximation schemes by means of a derivative expansion. In particular, we compare the results for the phase transition temperature, equation of state and the thermal mass of the scalar fields obtained in various approximations. We find that the large- $N_{c}$ approximation, being the zeroth order of our approximation, deviates significantly from approximation schemes in which effects of (Pseudo-) Goldstone modes have been taken into account, even at high temperatures and even if we allow for a finite explicit symmetry breaking. The important role of the (Pseudo-) Goldstone modes is also manifested in the phenomenon of "local order" which is missing in large- $N_{c}$ studies.

PACS numbers: 11.10.Wx,11.30.Rd,12.38.Aw,64.60.ae
\end{abstract}

\section{INTRODUCTION}

The thermodynamics of strongly interacting relativistic bosons and fermions play a prominent role in various fields of physics, such as heavy-ion collision experiments. There the theoretically computed equation of state of QCD is used as an input in order to describe the expansion of the so-called fireball [1]. However, the computation of the equation of state is a highly-involved problem since the high-temperature limit is not a weak-coupling limit, at least for the soft modes, see e. g. [2, 3]. On the contrary, by increasing the temperature we interpolate continuously between the $4 d$ theory and the underlying $3 d$ theory which does not generally have to be weakly coupled. Indeed, QCD in $3 d$ as well as simple scalar field theories in $3 d$ have shown to be strongly coupled. For this reason, a naive perturbative expansion of the partition function at high temperatures is bound to fail.

Several methods have been developed to study the equation of state. Lattice simulations are definitely the most powerful tool that we have currently at hand for a computation of the equation of state of strongly coupled theories. However, since lattice simulations are performed in a finite volume and at finite explicit symmetry breaking (i. e. current quark mass in the QCD context or external magnetic in the context of Ising models), the result for the equation of state has to be extrapolated to the continuum limit. The presence of dynamical fermions complicates lattice simulations further. On the other hand, continuum approaches have to deal with complementary problems, such as necessary truncations of the action of the theory.

One prominent approach to a systematic study of the thermodynamics of strongly interacting theories are hard-thermal loop resummation techniques, see e. g. Refs. [4, 5, 6, 7, 8, 9, 10, 11]. For example, it has been shown in this framework that the momentum dependence of the $n$-point functions longitudinal and transversal to the heat-bath has to be resolved carefully in order to properly describe the equation of state of hot QCD above the phase transition. On the other hand, low-energy models of QCD based on the NJL model [12] have been extensively used to study the thermodynamics of strongly interacting hadronic matter in the vicinity of the phase transition. However, these models are not confining and do not include gluodynamics. Therefore phenomenological extensions of these models have been proposed, see e. g. 13, 14, 15, 16, 17, 18, 19, 20]. By means of this, the thermodynamics at high temperatures are corrected in the sense that the missing gluon contributions are now incorporated. Still, the models are mostly studied in the limit of many colors - the large $N_{c}$ limit. As a consequence the contributions of the scalar fields, i. e. pions in QCD, are not properly taken into account. Neglecting the fluctuations of the scalar fields affects universal aspects of the theory in the vicinity of the phase transition, such as critical exponents, but also non-universal properties, such as the phase transition temperature or the equation of state.

In the present paper, our aim is to study systematically the role of the neglected fluctuations of the scalar fields and of the non-trivial momentum dependence of the propagators of the bosonic and fermionic fields. To this end, we apply a derivative expansion to a strongly interacting theory of bosons and fermions with $N_{c}$ colors but only one quark flavor. The low-temperature limit of our model is dominated by the presence of a single Goldstone boson (pion) rather than three as in QCD with two quark flavors. However, in the spirit of QCD low-energy models our model is as close as it can be to the large- $N_{c}$ approximation, where loops involving scalar fields are dropped. As we shall discuss below, the large- $N_{c}$ approximation represents the lowest order of our derivative expansion of the effective action. 
The paper is organized as follows: In Sect. II we introduce our model and the non-perturbative framework, namely the functional Renormalization Group (RG) 21], which we employ to compute the thermodynamic properties of our model. The results for the equation of state and the thermal mass of the scalar fields are then given in Sect. III. In addition we discuss the dependence of the equation of state on explicit symmetry breaking (i. e. current quark mass) and on the ultraviolet cutoff. Our concluding remarks, including a discussion of possible future extensions, are presented in Sect. IV.

\section{FUNCTIONAL RENORMALIZATION GROUP AND LOW-ENERGY MODELS}

\section{A. The model and the studied truncations}

Throughout this paper we work in $d=4$ dimensional Euclidean space and employ the following ansatz for the effective action for a strongly-interacting theory of bosons and fermions:

$$
\begin{array}{r}
\Gamma=\int d^{4} x\left\{Z_{\psi} \bar{\psi}_{a} \mathrm{i} \not \partial \psi_{a}+\frac{\bar{h}}{\sqrt{2}} \bar{\psi}_{a}(\vec{\tau} \cdot \Phi) \psi_{a}\right. \\
\left.+\frac{1}{2} Z_{\phi}\left(\partial_{\mu} \Phi\right)^{2}+U\left(\Phi^{2}\right)\right\},
\end{array}
$$

where the index of the fermion fields denotes an internal index, e. g. a color index in QCD, and runs from $a=1, \ldots, N_{c}$. The scalar fields are combined in the $O(2)$ vector $\Phi^{T}=(\sigma, \pi)$ and we have used $\vec{\tau}=\left(\gamma_{5}, \mathrm{i} \cdot \mathbf{1}\right)$ in order to define a chirally invariant Yukawa interaction. We assume that the bosons are composite degrees of fermions and do not carry an internal charge, e. g. color: $\sigma \sim \bar{\psi}_{a} \psi_{a}$ and $\pi \sim \bar{\psi}_{a} \gamma_{5} \psi_{a}$. In the spirit of hadronic models, our ansatz for the effective action represents a low-energy model for QCD with one quark flavor. However, we rush to add that our interest is not the study of one-flavor QCD. In this case, we would have to take the effects of topologically non-trivial gauge configurations into account which are prominent in one-flavor QCD since they induce mass-like fermion interactions and break the $U_{A}(1)$ symmetry [22, 23, 24, 25]. Here, we are interested in an investigation of the effect of light bosonic degrees of freedom on the thermodynamics. In this respect, our model with only one dynamical Goldstone mode allows us to mark out the differences to large $N_{c}$ studies of presently used QCD low-energy models in a simple way. Note that the Goldstone-mode effects become even more pronounced with increasing number of quark flavors since the number of Goldstone modes rises quadratically with the number of quark flavors.

Let us now discuss the various truncations which we use to study the phase transition temperature and the thermodynamics of our model. Our model is built around the standard mean-field ansatz for the effective action

\begin{tabular}{l|l}
\hline \hline Label & Approximation \\
\hline large $N_{c}$ & drop bosonic loops \& $\partial_{t} Z_{\phi}^{\perp} \equiv 0$ \\
LPA & $\partial_{t} Z_{\phi}^{\perp} \equiv 0, Z_{\phi}^{\|} \equiv Z_{\phi}^{\perp} \& \partial_{t} Z_{\psi}^{\perp} \equiv 0, Z_{\psi}^{\|} \equiv Z_{\psi}^{\perp}$ \\
Trunc. A & $\partial_{t} Z_{\phi}^{\perp} \neq 0, Z_{\phi}^{\|} \equiv Z_{\phi}^{\perp} \& \partial_{t} Z_{\psi}^{\perp} \equiv 0, Z_{\psi}^{\|} \equiv Z_{\psi}^{\perp}$ \\
Trunc. B & $\partial_{t} Z_{\phi}^{\perp} \neq 0, Z_{\phi}^{\|} \equiv Z_{\phi}^{\perp} \& \partial_{t} Z_{\psi}^{\perp} \neq 0, Z_{\psi}^{\|} \equiv Z_{\psi}^{\perp}$ \\
Trunc. C & $\partial_{t} Z_{\phi}^{\perp} \neq 0, \partial_{t} Z_{\phi}^{\|} \neq 0 \& \partial_{t} Z_{\psi}^{\perp} \neq 0, \partial_{t} Z_{\psi}^{\|} \neq 0$ \\
\hline
\end{tabular}

Table I: Specification of the various truncations used to compute the equation of state.

(large- $N_{c}$ ansatz), i. e.

$$
Z_{\phi} \equiv 0 \quad \text { and } \quad Z_{\psi} \equiv 1
$$

Such an ansatz has been used extensively for studies of the QCD phase diagram, see e. g. Refs. [26, 27] and underlies also most of the recent (P)NJL studies of hot and dense QCD, see e. g. Refs. [15, 17, 18, 19, 20].

In the following we systematically extend this zerothorder ansatz in two directions, namely in the number of derivatives and the number $n$ of external legs of $n$-point functions $\Gamma^{(n)}$. In order to study spontaneous symmetry breaking which is indicated by a non-trivial minimum of the order-parameter potential $U\left(\Phi^{2}\right)$ in Eq. (1), we expand the potential in powers of $\Phi^{2}$. This results in RG flow equations for the mesonic $n$-point functions. On the other hand, we perform a derivative expansion which renders the $n$-point functions momentum dependent. Note that the latter is indispensable for a connection of the high- and low-momentum regime of QCD [28, 29, 30], see also Refs. 31, 32.

Next to the zeroth-order approximation (large $N_{c}$ ) one needs to include kinetic terms for the meson fields. The minimal truncation which allows for an inclusion of meson loops is given by the so-called Local Potential Approximation (LPA), i. e.

$$
Z_{\phi} \equiv 1 \quad \text { and } \quad Z_{\psi} \equiv 1
$$

This truncation has been used, e. g., in Refs. [16, 33, 34, 35] for a study of the quark-meson model at finite temperature and density. It turns out that the LPA represents indeed already a major improvement with respect to the quality of the critical exponents. At this point, we would like to remark that the quality of critical exponents can be considered as a measure of how well the dynamics at the phase transition are captured.

Next to LPA, we consider a truncation in which we allow for a running of the scalar wave-function renormalization, but keep the wave-function renormalization of the fermions fixed, i. e.

$$
\begin{array}{cc}
Z_{\phi}(k=\Lambda) \ll 1, & \partial_{t} Z_{\phi} \neq 0, \\
Z_{\psi}(k=\Lambda)=1, & \partial_{t} Z_{\psi} \equiv 0,
\end{array}
$$

where $t=\ln (k / \Lambda)$ and $k$ denotes the IR (infrared) cutoff scale of our functional RG approach and $\Lambda$ denotes the 
UV (ultraviolet) cutoff, see Subsec. 【B for details. By means of this approximation, vertices involving at least one boson field become momentum dependent. Thereby the quality of the results is already improved considerably which can be read off from the quality of the critical exponents $^{1}$, see e. g. Refs. [37, 38, 39]. We shall denote this as truncation A from now on.

In our truncation $\mathrm{B}$, we go beyond this approximation and allow for a running of the wave-function renormalizations of the fermion fields as well, i. e.

$$
\begin{array}{cc}
Z_{\phi}(k=\Lambda) \ll 1, & \partial_{t} Z_{\phi} \neq 0, \\
Z_{\psi}(k=\Lambda)=1, & \partial_{t} Z_{\psi} \neq 0 .
\end{array}
$$

Such a truncation renders all vertices momentum dependent.

At finite temperature the wave-function renormalizations longitudinal $(\|)$ and transversal $(\perp)$ to the heatbath obey in general a different RG running. In order to study this difference, we have to replace the part of the truncation (1) involving derivatives according to

$$
\begin{aligned}
& Z_{\psi} \bar{\psi}_{a} \mathrm{i} \not \partial \psi_{a}+\frac{1}{2} Z_{\phi}\left(\partial_{\mu} \Phi\right)^{2} \\
& \longrightarrow Z_{\psi}^{\|} \bar{\psi}_{a} \mathrm{i} \gamma_{0} \partial_{0} \psi_{a}+\frac{1}{2} Z_{\phi}^{\|}\left(\partial_{0} \Phi\right)^{2} \\
& \quad+Z_{\psi}^{\perp} \bar{\psi}_{a} \mathrm{i} \gamma_{i} \partial_{i} \psi_{a}+\frac{1}{2} Z_{\phi}^{\perp}\left(\partial_{i} \Phi\right)^{2},
\end{aligned}
$$

where we identify $Z_{\psi, \phi}^{\perp}$ with $Z_{\psi, \phi}$ in both truncation A and B but allow for a different running of $Z_{\psi, \phi}^{\perp}$ and $Z_{\psi, \phi}^{\|}$. We shall denote this as truncation $\mathrm{C}$. The various truncations alongside with their labels are briefly summarized in Tab. [1.

As mentioned above, we expand the scalar potential $U\left(\Phi^{2}\right)$ (order-parameter potential) in a power series of $\Phi^{2}$ up to order $\Phi^{4}$ and drop all higher terms [40, 41, 42]:

$$
\begin{aligned}
U\left(\Phi^{2}\right) \equiv U(\rho, \sigma) & =\sum_{\alpha=0}^{\infty} \frac{\bar{\lambda}_{\alpha}}{\alpha !}\left(\rho-\rho_{0}\right)^{\alpha}-c \sigma \\
& \approx \bar{\lambda}_{0}+\bar{\lambda}_{1}\left(\rho-\rho_{0}\right)+\frac{\bar{\lambda}_{2}}{2}\left(\rho-\rho_{0}\right)^{2}-c \sigma
\end{aligned}
$$

where $\rho=(1 / 2) \Phi^{2}, \Phi_{0}^{T}=\left(\sigma_{0}, 0\right)$ and the parameter $c$ is a measure for the strength of explicit symmetry breaking. Note that such an expansion represents a systematic expansion in $n$-point functions $\Gamma^{(n)}$ where $n=2 \alpha$ defines the number of external legs and is well under control. In fact, the quality of an expansion of $U\left(\Phi^{2}\right)$ in powers of $\Phi^{2}$ has been studied quantitatively in Ref. [37] at vanishing temperature and for the proper-time RG in LPA

\footnotetext{
1 The derivative expansion can be continued systematically by e. $\mathrm{g}$. including terms of the form $Y_{k}\left(\Phi \partial_{\mu} \Phi\right)^{2}$ in our truncation [36].
}

at finite temperature in Ref. [43]. In Ref. [34], the orderparameter potential $U$ has been computed in LPA for a quark-meson model with two quark flavors within a RG approach without making use of a Taylor expansion in $\Phi$. Note that we also solve the RG flow in the large- $N_{c}$ approximation by making use of the expansion (3) of the order-parameter potential $U$, even though it is straightforward to solve the flow in this case without truncating the power series in Eq. (3), see e. g. Ref. [26]. In our case a Taylor expansion of the potential is justified since (i) we want to focus on the role of the momentum dependence of the vertices and (ii) have to ensure comparability of our results from our various truncations.

From the condition $\partial U / \partial \sigma=0$ evaluated at $\sigma=\sigma_{0}$, we find that the RG flow of the coupling $\bar{\lambda}_{1}$ and the minimum $\sigma_{0}(k)$ are related:

$$
\bar{\lambda}_{1}(k) \sigma_{0}(k)=c .
$$

This condition keeps the minimum at $(\sigma, \pi)=\left(\sigma_{0}(k), 0\right)$. The flow equation of the minimum $\sigma_{0}(k)$ is thus related to the flow of the coupling $\bar{\lambda}_{1}$ in a simple way. The initial conditions for the various couplings in Eq. (11) at the ultraviolet scale $\Lambda$ are discussed in Sec. III.

\section{B. RG flow at finite temperature}

For our derivation of the RG flow equations of the couplings, we employ the Wetterich equation [21]:

$$
\partial_{t} \Gamma_{k}[\chi]=\frac{1}{2} \operatorname{STr}\left\{\left[\Gamma_{k}^{(1,1)}[\chi]+R_{k}\right]^{-1} \cdot\left(\partial_{t} R_{k}\right)\right\}
$$

with

$$
\Gamma_{k}^{(1,1)}[\chi]=\frac{\vec{\delta}}{\delta \chi^{T}} \Gamma_{k}[\chi] \frac{\overleftarrow{\delta}}{\delta \chi}
$$

where $t=\ln k / \Lambda$ and $\Lambda$ is the UV cutoff scale. Here, $\chi$ represents a vector in field space and is defined as

$$
\chi^{T} \equiv \chi^{T}(-q):=\left(\psi^{T}(-q), \bar{\psi}(q), \Phi_{1}(-q), \Phi_{2}(-q)\right)
$$

and

$$
\chi \equiv \chi(q):=\left(\begin{array}{c}
\psi(q) \\
\bar{\psi}^{T}(-q) \\
\Phi_{1}(q) \\
\Phi_{2}(q)
\end{array}\right) .
$$

Thus, $\Gamma_{k}^{(1,1)}[\chi]$ is matrix-valued in field space and so is the regulator function $R_{k}$. In this work, we employ a $3 d$ optimized regulator function which is technically advantageous for studies at finite temperature [16, 44, 45]. The quality of such a $3 d$ regulator in the limit of vanishing temperature and chemical potential has been estimated by computing critical exponents of $O(N)$ models [46] and 
comparing them to those optained with an optimized regulator in $4 d$ space-time [47, 48]. Details on the regularization can be found in App. B. In the following we give the RG flow equations in a way which does not dependent on the details of our $3 d$ regularization. Reviews on and introductions to the functional RG and its application to gauge theories can be found in Refs. [29, 39, 49, 50, 51].

Inserting our ansatz for the effective action (1) into the flow equation (6), we find the flow equation for the order-parameter potential $U$ :

$$
\begin{aligned}
\partial_{t} U=\frac{8 v_{3}}{3} k^{4}\left\{\frac{\hat{z}_{\phi}^{-1 / 2}}{\sqrt{1+m_{\sigma}^{2}}}\left(\frac{1}{2}+n_{B}\left(\hat{z}_{\phi}, m_{\sigma}\right)\right)\right. \\
+\frac{\hat{z}_{\phi}^{-1 / 2}}{\sqrt{1+m_{\pi}^{2}}}\left(\frac{1}{2}+n_{B}\left(\hat{z}_{\phi}, m_{\pi}\right)\right) \\
\left.-\frac{2 N_{c} \hat{z}_{\psi}^{-1}}{\sqrt{1+m_{q}^{2}}}\left(\frac{1}{2}-n_{F}\left(\hat{z}_{\psi}, m_{\psi}\right)\right)\right\},
\end{aligned}
$$

where $v_{3}=1 /\left(8 \pi^{2}\right), \hat{z}_{\phi}=Z_{\phi}^{\|} / Z_{\phi}^{\perp}, \hat{z}_{\psi}=Z_{\psi}^{\|} / Z_{\psi}^{\perp}$ and

$$
\begin{aligned}
& n_{B}(\hat{z}, m)=\left(\mathrm{e}^{\frac{\sqrt{k^{2}+m^{2}}}{\hat{z}^{1 / 2} T}}-1\right)^{-1} \\
& n_{F}(\hat{z}, m)=\left(\mathrm{e}^{\frac{\sqrt{k^{2}+m^{2}}}{\hat{z} T}}+1\right)^{-1}
\end{aligned}
$$

denote the Bose-Einstein and Fermi-Dirac distribution function, respectively. The renormalized masses of the bosons and fermions are given by

$$
\begin{aligned}
& m_{\sigma}^{2}=\frac{1}{Z_{\phi}^{\perp} k^{2}}\left(\frac{\partial U}{\partial \rho}+2 \rho \frac{\partial^{2} U}{\partial \rho^{2}}\right), \\
& m_{\pi}^{2}=\frac{1}{Z_{\phi}^{\perp} k^{2}}\left(\frac{\partial U}{\partial \rho}\right), \\
& m_{\psi}^{2}=\frac{\bar{h}^{2} \rho}{\left(Z_{\psi}^{\perp}\right)^{2} k^{2}}=h^{2}\left(\frac{Z_{\phi}^{\perp} \rho}{k^{2}}\right) .
\end{aligned}
$$

Here we have introduced the renormalized Yukawa coupling $h=\bar{h} /\left(Z_{\psi}^{\perp}\left(Z_{\phi}^{\perp}\right)^{1 / 2}\right)$. Note that the large- $N_{c}$ limit can be deduced from Eq. (17) by dropping the bosonic loops as well as the running of the wave-function renormalizations.

In Eq. (7) and (9) we observe that the ratio of the wavefunction renormalizations $\hat{z}_{\phi}=Z_{\phi}^{\|} / Z_{\phi}^{\perp}$ and $\hat{z}_{\psi}=Z_{\psi}^{\|} / Z_{\psi}^{\perp}$ effectively rescale the (dimensionless) temperature $\tilde{t}=$ $T / k$ in the loop diagrams.

The anomalous dimensions,

$$
\eta_{\phi}^{\|, \perp}=-\partial_{t} \ln Z_{\phi}^{\|, \perp}
$$

associated with the scalar wave-function renormalizations longitudinal $(\|)$ and transversal $(\perp)$ to the heat- bath are given by

$$
\begin{gathered}
\eta_{\phi}^{\perp}=\frac{4 v_{3}}{3} N_{c}\left[h^{2} \mathcal{M}_{4, \perp}^{(F)}\left(\tilde{t}, m_{\psi}^{2}\right)+4 \kappa h^{4} \mathcal{M}_{2, \perp}^{(F)}\left(\tilde{t}, m_{\psi}^{2}\right)\right. \\
\left.+4 \frac{\kappa \lambda_{\phi}^{2}}{N_{c}} \mathcal{M}_{2,2, \perp}^{(B)}\left(\tilde{t}, m_{\sigma}^{2}, m_{\pi}^{2}\right)\right], \\
\eta_{\phi}^{\|}=\frac{4 v_{3}}{\hat{z}_{\phi}} N_{c}\left[h^{2} \mathcal{M}_{4, \|}^{(F)}\left(\tilde{t}, m_{\psi}^{2}\right)+4 \kappa h^{4} \mathcal{M}_{2, \|}^{(F)}\left(\tilde{t}, m_{\psi}^{2}\right)\right. \\
\left.+2 \frac{\kappa \lambda_{\phi}^{2}}{N_{c}} \mathcal{M}_{2,2, \|}^{(B)}\left(\tilde{t}, m_{\sigma}^{2}, m_{\pi}^{2}\right)\right],
\end{gathered}
$$

where $\kappa=Z_{\phi}^{\perp} \rho_{0} / k^{2}$ and $\lambda_{\phi}=\bar{\lambda}_{2} /\left(Z_{\phi}^{\perp}\right)^{2}$. The anomalous dimensions,

$$
\eta_{\psi}^{\|, \perp}=-\partial_{t} \ln Z_{\psi}^{\|, \perp},
$$

of the fermion fields read

$$
\begin{aligned}
& \eta_{\psi}^{\perp}=\frac{4 v_{3}}{3} h^{2}\left[\mathcal{M}_{1,2, \perp}^{(F B)}\left(\tilde{t}, m_{\psi}^{2}, m_{\pi}^{2}\right)\right. \\
&\left.+\mathcal{M}_{1,2, \perp}^{(F B)}\left(\tilde{t}, m_{\psi}^{2}, m_{\sigma}^{2}\right)\right], \\
& \eta_{\psi}^{\|}=\frac{4 v_{3}}{\hat{z}_{\psi}} h^{2}\left[\mathcal{M}_{1,2, \|}^{(F B)}\left(\tilde{t}, m_{\psi}^{2}, m_{\pi}^{2}\right)\right. \\
&\left.\quad+\mathcal{M}_{1,2, \|}^{(F B)}\left(\tilde{t}, m_{\psi}^{2}, m_{\sigma}^{2}\right)\right] .
\end{aligned}
$$

The threshold functions $\mathcal{M}$ are defined in Ref. [30] and App. B and describe the decoupling of massive and thermal modes. The $\mathrm{RG}$ flows of the ratios $\hat{z}_{\phi}=Z_{\phi}^{\|} / Z_{\phi}^{\perp}$ and $\hat{z}_{\psi}=Z_{\psi}^{\|} / Z_{\psi}^{\perp}$ read

$$
\begin{aligned}
& \hat{\eta}_{\phi}=-\frac{\partial_{t} \hat{z}_{\phi}}{\hat{z}_{\phi}}=\eta_{\phi}^{\|}-\eta_{\phi}^{\perp}, \\
& \hat{\eta}_{\psi}=-\frac{\partial_{t} \hat{z}_{\psi}}{\hat{z}_{\psi}}=\eta_{\psi}^{\|}-\eta_{\psi}^{\perp} .
\end{aligned}
$$

Since $\eta_{\phi}^{\|}=\eta_{\phi}^{\perp}$ and $\eta_{\psi}^{\|}=\eta_{\psi}^{\perp}$ for $T / k=\tilde{t}=0$, which is a property of the threshold functions $\mathcal{M}$, we have $\hat{\eta}_{\phi}=0$ for $\tilde{t}=0$ as expected for a Poincare-invariant theory.

The RG flow of the Yukawa coupling can be calculated along the lines of Ref. [30]. We find

$$
\begin{gathered}
\partial_{t} h^{2}=\left(\eta_{\phi}^{\perp}+2 \eta_{\psi}^{\perp}\right) h^{2}-4 v_{3} h^{4}\left[L_{1,1}^{(F B)}\left(m_{\psi}^{2}, 0, m_{\pi}^{2}\right)\right. \\
\left.-L_{1,1}^{(F B)}\left(\tilde{t}, m_{\psi}^{2}, 0, m_{\sigma}^{2}\right)\right]
\end{gathered}
$$

The threshold function $L_{1,1}^{(F B),(4)}$ can be found in Ref. [30]. In the limit $\tilde{t}=T / k \rightarrow 0$, we recover the perturbative result $\partial_{t} h^{2}=\left(\left(N_{c}+1\right) /\left(8 \pi^{2}\right)\right) h^{4}$, see e. g. Ref. [28]. In the chirally symmetric regime $\left(m_{\sigma}=m_{\pi}\right)$, we find that the RG flow of the Yukawa coupling is governed by the IR attractive fixed point $h^{*}=0$ for $\tilde{t}=T / k \rightarrow 0$. For $\tilde{t} \rightarrow \infty$, we have $\beta_{h^{2}} \equiv \partial_{t} h^{2} \rightarrow 0$ which is due to the decoupling of the fermions for $k \lesssim T$. Note that it is special to our model with just one fermion species $\left(N_{f}=1\right)$ that the running of the Yukawa coupling 
is only driven by the anomalous dimensions in the symmetric regime. For example, in QCD with more than one quark flavor we would find additional contributions to the RG flow of the Yukawa coupling since the contributions from the Goldstone modes in the symmetric regime are not canceled completely by the ones of the radial mode [52, 53]. In the regime with broken $O(2)$ symmetry in the ground state, these additional terms emerge when $m_{\sigma} \neq m_{\pi}$.

Let us close this section with some general remarks on the RG flow of $Z_{\psi, \phi}^{\|}$and $Z_{\psi, \phi}^{\perp}$ at vanishing temperature. As mentioned above, we employ a $3 d$ regulator function in order to derive the flow equations. Thus, our regularization breaks necessarily the $O(d)$ symmetry in the derivative terms of our truncation, i. e. $\partial_{t} Z_{\psi, \phi}^{\|} \neq \partial_{t} Z_{\psi, \phi}^{\perp}$ even for $T / k \rightarrow 0$. In LPA and in large- $N_{c}$ approximations, this problem does not appear since the nontrivial momentum dependence of the propagators is neglected [16, 34, 44, 45]. In studies beyond LPA, we have to deal with the broken Poincare-invariance at vanishing temperature due to the choice of our $3 d$ regulator function $^{2}$. In principle one can solve this problem by taking care of the symmetry violating terms with the aid of the corresponding Ward identities. Equivalently, we can choose the initial conditions for the RG flow equations such that one finds $Z_{\psi, \phi}^{\perp}=Z_{\psi, \phi}^{\|}$for $k \rightarrow 0$ and $T \rightarrow 0$. In the present paper, we adjust the initial conditions properly in order to deal with this circumstance ${ }^{3}$. At finite temperature, the breaking of the $O(d)$ symmetry in momentum space due to our choice of the regulator function does not harm our study since this symmetry is broken anyway. However, the choice of our regulator function allows us to perform the Matsubara sums analytically, which simplifies the numerics considerably and justifies our choice for the regulator function.

\section{EQUATION OF STATE AND THERMAL MASS}

Let us first discuss our choice for the initial conditions. Since we are interested in studying how the momentum dependence of the propagators and vertices affects the phase-transition temperature and the thermodynamic pressure, we adjust the parameters (initial conditions at the UV scale $\Lambda$ ) of the model such that we obtain the same values for the IR observables for all truncations in this work. To be specific, we choose $\Lambda=2 \mathrm{GeV}$ and $f_{\pi} \equiv \sqrt{\rho_{0}}=0.030 \mathrm{GeV}, m_{q}=0.088 \mathrm{GeV}$ and $Z_{\psi, \phi}^{\perp}=Z_{\psi, \phi}^{\|}$at $k=0$ and $T=0$. Moreover we use

\footnotetext{
2 This issue does not occur if one applies a $4 d$ regulator function.

${ }^{3}$ From a field-theoretical point of view the adjustment of the initial conditions of a given truncation means nothing else than adding appropriate counter-terms such that the theory remains Poincare-invariant for $k \rightarrow 0$ and $T \rightarrow 0$.
}

\begin{tabular}{l|c|c|c|c}
\hline \hline Truncation & $T_{\mathrm{c}} / f_{\pi}$ & $T_{\max } / f_{\pi}$ & $T_{\max } / T_{\mathrm{c}}$ & $k_{\chi \mathrm{SB}}^{T=0} / f_{\pi}$ \\
\hline large $N_{c}$ & 2.87 & - & - & 8.96 \\
LPA & 2.12 & 2.35 & 1.11 & 8.93 \\
Trunc. A & 1.96 & 2.12 & 1.08 & 8.24 \\
Trunc. B & 2.06 & 2.26 & 1.10 & 8.85 \\
Trunc. C & 1.93 & 2.12 & 1.09 & 8.78 \\
\hline \hline
\end{tabular}

Table II: Results for the phase transition temperature $T_{\mathrm{c}}$, the position of the maximum in the pressure, $T_{\max }$, and the chiral symmetry breaking scale $k_{\chi \mathrm{SB}}$ at vanishing temperature. All values are given in units of the value of the order parameter $f_{\pi}=\sqrt{\rho_{0}}=\sigma_{0} / \sqrt{2}=30 \mathrm{MeV}$ at vanishing temperature.

$N_{c}=3$ in the numerical evaluation of the RG flow equations. Our choice for the parameters ensures that (i) the UV cutoff $\Lambda$ is much larger than the physically relevant scales in order to avoid cutoff effects in the hightemperature regime ${ }^{4}\left(T>T_{\mathrm{c}}\right)$ and (ii) that dimensionless ratios of observables are close to the ones in QCD with $N_{f}=2$ and $N_{f}=2+1$ quark flavors. Indeed, our choice for the parameters yields $m_{q} / f_{\pi} \approx 3$. Since the actual dimensionful values for the critical temperature is of no interest in this paper, we give all results in units of $f_{\pi}$. This enables us to compare the results from the various truncations in a simple manner.

The results for the phase-transition temperature $T_{\mathrm{c}}$ for the various truncations are listed in Tab. III We observe that the phase transition temperature decreases when we take bosonic loops into account and resolve the momentum dependence of the vertices. Since the Goldstone boson plays a prominent role at the phase transition, it is intuitively clear that the inclusion of these effects affect the dynamics near the phase transition. Moreover, Goldstone bosons tend to restore the symmetry while fermions tend to build up a condensate and thereby break the symmetry of the ground state. However, the anti-periodic boundary conditions for the fermions in Euclidean time direction lead to a suppression of the fermionic modes in the vicinity and above the phase transition due to the absence of a zero mode.

In Fig. 1 the influence of the bosonic fluctuations is visible in a different observable, namely the behavior of the order parameter near the phase transition which can be measured in terms of a universal critical exponent $\beta$, $f_{\pi} \sim\left|T-T_{\mathrm{c}}\right|^{\beta}$. We observe that the slope of the order parameter at the phase transition found in our large- $N_{c}$ study deviates clearly from the slope found in truncations including bosonic fluctuations. For example, we

\footnotetext{
${ }^{4}$ While the UV cutoff $\Lambda$ can be chosen almost arbitrarily in LPA and in our large- $N_{c}$ approximation, the possible choices for the initial conditions (UV parameters) for a given set of IR observables in truncations beyond LPA is restricted by the running of the Yukawa coupling. In this sense, these models have some predictive power even at vanishing temperature [52].
} 


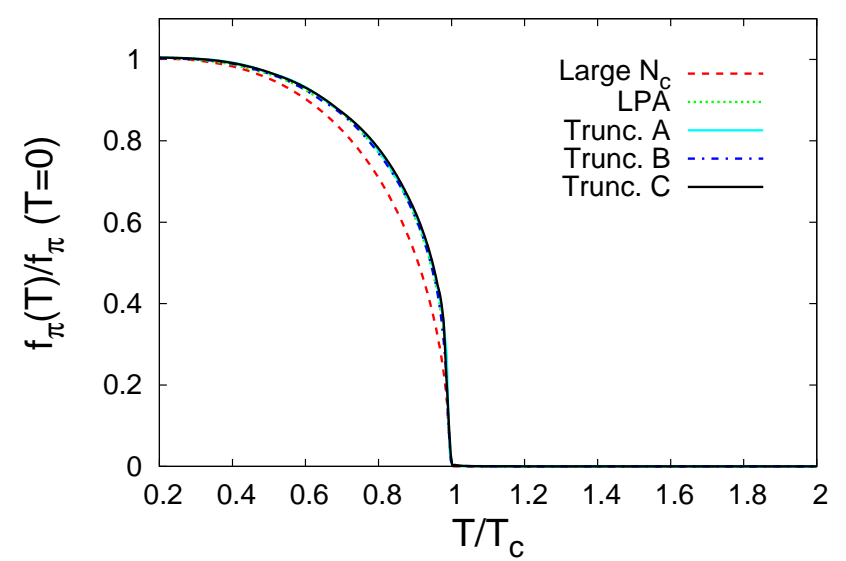

Figure 1: (Color online) Order parameter divided by its zerotemperature value as a function of $T / T_{\mathrm{c}}$ as obtained from the various different truncations for the momentum dependence. For all truncations beyond large- $N_{c}$, the results for the order parameter are indistinguishable on the shown resolution. The different shape of the large- $N_{c}$ result is due to the fact that the value of the corresponding critical exponent differs from the exact value.

find $\beta_{\text {large } N_{c}}=0.500 \pm 0.001$ and $\beta_{\mathrm{LPA}}=0.349 \pm 0.011$. Our large- $N_{c}$ value agrees nicely with the analytically known value, $\beta_{\text {large } N_{c}}^{\text {exact }}=1 / 2$. The LPA value is in agreement with the corresponding values found in Refs. [54] and [37]. The error bars arise from a fit to our numerical data for the order parameter.

Next we discuss our results for the pressure. The flow equation for the pressure is obtained from the negated right-hand side of the flow equation (7) for the orderparameter potential evaluated at the physical ground state $\Phi_{0}$. In Fig. 2, we show our results for the pressure scaled by $T^{4}$ as a function of $T / T_{\mathrm{c}}$ as obtained from LPA as well as from the truncations A-C; we shall discuss the large- $N_{c}$ limit below. Since we are considering the limit of vanishing external linear symmetry breaking (chiral limit, $c \rightarrow 0$ in Eq. (3)), we observe that the pressure approaches the Stefan-Boltzmann (SB) limit of one massless boson for small temperatures, $p / T^{4} \rightarrow \pi^{2} / 90$ for $T \rightarrow 0$. When we increase the temperature starting from $T=0$, we find that the pressure increases since the mass of the fermions, $m_{q}=h f_{\pi}$, decreases, see also Fig. 1. At $T=T_{\mathrm{c}}$ the fermions become massless while the bosons acquire a thermal mass $m_{\phi}=m_{\sigma}=m_{\pi}$. However, the pressure neither takes its maximal value at $T_{\mathrm{c}}$ nor does it agree with the Stefan-Boltzmann limit of a free gas of massless fermions. On the contrary, we observe that the pressure function reaches a maximum at $T_{\max }>T_{\mathrm{c}}$ and approaches the Stefan-Boltzmann limit of a gas of free massless quarks for $T>T_{\max }$ slowly from above. We would like to stress that this is a substantial difference to the findings in a large- $N_{c}$ approximations as we shall see below. At $T=T_{\max }$, we find

$$
\left.\frac{p}{T^{4}}\right|_{T=T_{\max }} \approx 4 N_{c} \frac{7}{8} \frac{\pi^{2}}{90}+2 \frac{\pi^{2}}{90}
$$

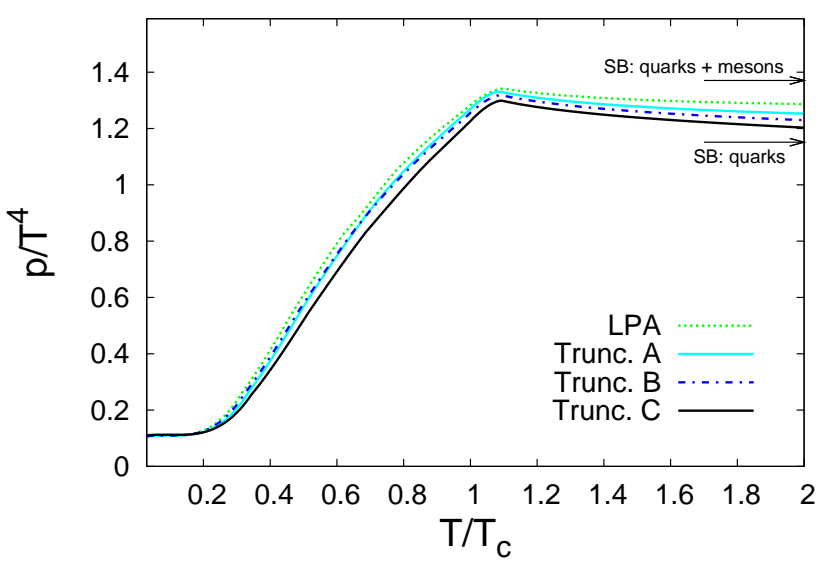

Figure 2: (Color online) Pressure as a function of $T / T_{\mathrm{c}}$ as obtained from the different truncations of the momentum dependence of the propagators and vertices. We observe a maximum in the pressure function located slightly above $T_{\mathrm{c}}$. For high temperatures, we observe that the Stefan-Boltzmann (SB) limit of a free gas of fermions (quarks) is approached from above.

which is the pressure of a gas of $4 N_{c}$ massless fermions and 2 massless bosons. This result can be easily generalized to the case with $N_{f}$ fermion species. We expect that the maximal pressure of a QCD low-energy model with $N_{f} \geq 2$ quark flavors is given by ${ }^{5}$

$$
\begin{aligned}
& \left.\frac{p}{T^{4}}\right|_{T_{\max }, N_{f} \geq 2} \approx 4 N_{c} N_{f} \frac{7}{8} \frac{\pi^{2}}{90}+N_{f}^{2} \frac{\pi^{2}}{90} \\
& \left.\Rightarrow \frac{p}{p_{\text {free quarks }}}\right|_{T=T_{\max }, N_{f} \geq 2} \approx 1+\frac{2}{7} \frac{N_{f}}{N_{c}} .
\end{aligned}
$$

In the second line we have divided our estimate for the ratio of the pressure at $T=T_{\max }$ with the StefanBoltzmann limit of a gas of free massless quarks.

The observation that $T_{\max }>T_{\mathrm{c}}$ reflects the fact that chiral symmetry is not restored on all scales of the RG flow for $T_{\mathrm{c}} \leq T \leq T_{\max }$ but broken on intermediate scales $k>0$. This is depicted in Fig. 3 where we show the pion decay constant $f_{\pi}$, i. e. the order parameter, as a function of the RG scale $k$ in the temperature regime $T_{\mathrm{c}} \leq T \leq T_{\max }$. The behavior of $f_{\pi}$ displays the interplay of the bosons and fermions in the RG flow: The fermions dominate the dynamics in the RG flow and tend to break chiral symmetry for $k / T \gg 1$. Below the chiral symmetry breaking scale $k_{\chi \mathrm{SB}}$ the fermions acquire then a mass and therefore decouple from the flow. Moreover all fermionic modes acquire a thermal mass $\sim T$. In contradistinction to the fermions, the bosonic fields have a thermal zero mode. Thus the RG flow is governed by the Goldstone boson for $k / T \lesssim 1$ which drives the system

\footnotetext{
${ }^{5}$ Our observation of the existence of a maximum in the pressure function is in accordance with an estimate for the pressure function in Ref. 33] obtained from studying a gas of non-interacting quarks and mesons with temperature-dependent masses.
} 


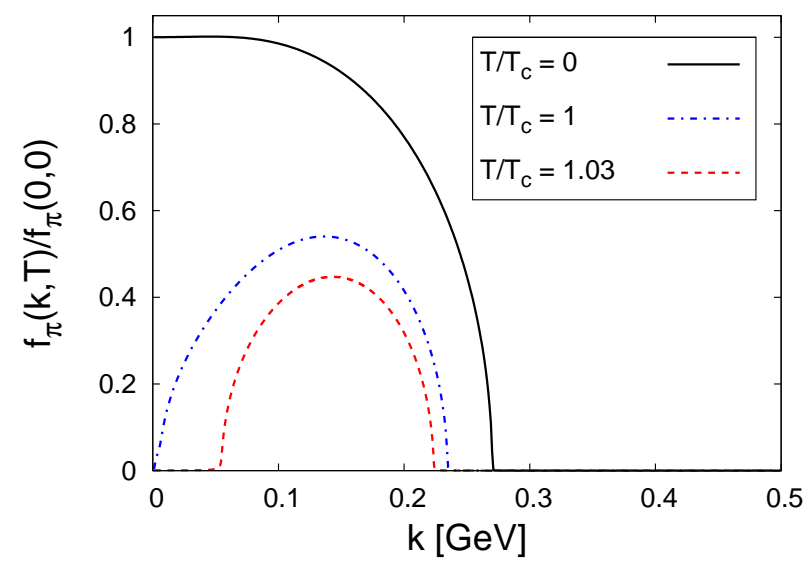

Figure 3: (Color online) RG flow of the pion decay constant in LPA for $T / T_{\mathrm{c}}=0,1,1.03$. We observe that a regime exists in which the pion decay constant is finite even though the system is the phase with restored chiral symmetry for $k \rightarrow 0$. Note that the behavior of $f_{\pi}$ is qualitatively the same for the truncations A-C.

towards the regime with restored chiral symmetry. Since the IR regulator scale $k$ introduces an intrinsic length scale $\sim 1 / k$, we conclude that we have "local order" for $T_{\mathrm{c}} \leq T \leq T_{\max }$, i. e. the system is ordered on spatial domains ${ }^{6}$ of length scales $\sim 1 / k$. This implies that the bosons contribute still significantly to the pressure for these temperatures. On the other hand, the fermions give a significant contribution as well in this regime since their mass is zero for $k \rightarrow 0$. For $T>T_{\max }$, the system remains in the symmetric regime on all RG scales. Thus we have "global disorder" for these temperatures. Let us further discuss this phenomenon: In a Landau-Ginzburg type description of phase transitions, symmetry breaking is indicated by a negative squared mass parameter (i. e. by a finite vacuum expectation value of the orderparameter potential). In an RG approach this parameter is scale-dependent. Usually we are only interested in the shape of the potential in the limit $k \rightarrow 0$. For $T>T_{\max }$ the squared mass parameter of the Landau-Ginzburg potential remains positive on all scales. On the other hand, the squared mass parameter becomes negative on intermediate scales for $T_{\mathrm{c}} \leq T \leq T_{\max }$ but becomes positive again in the physical limit $k \rightarrow 0$, see also Fig. 3 where the regimes of positive mass squared correspond to a vanishing order parameter. Below the phase transition temperature $\left(T<T_{\mathrm{c}}\right)$ the squared mass parameter becomes negative at the chiral symmetry breaking scale $k_{\chi \mathrm{SB}}$ and stays negative even for $k \rightarrow 0$.

Note that $T_{\mathrm{c}}$ and $T_{\max }$ differ only by roughly $10 \%$, see Tab. [II Therefore the phase transition temperature can indeed be estimated reasonably well by just seeking for

\footnotetext{
${ }^{6}$ We would like to remark that the inverse IR regulator scale $1 / k$ can be roughly associated with the side length $L$ of the spatial simulation volume in lattice QCD simulations, see also Ref. [55].
}

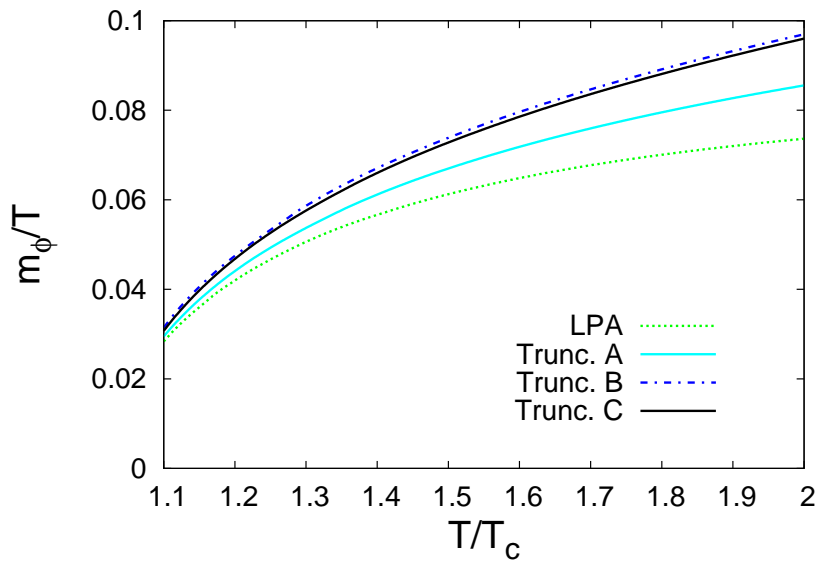

Figure 4: (Color online) Thermal mass $m_{\phi}$ of the scalar fields divided by the temperature as a function of $T / T_{\mathrm{c}}$ as obtained from the different truncations for the momentum dependence. The increase of the thermal mass goes alongside with the decrease of the pressure function for the shown temperatures.

the temperature for which the squared mass parameter of a Landau-Ginzburg-type potential stays positive on all scales. This strategy has been applied in Refs. [56, 57] in order to estimate the chiral phase transition in QCD from RG flows involving gluonic degrees of freedom.

In Fig. 2 and Tab. [II, we also observe that the position of the maximum of the pressure is almost independent of the truncation. For $T>T_{\max }$ we find that the StefanBoltzmann limit of a free gas of massless quarks is approached faster for truncations in which the momentum dependence of the vertices has been resolved more accurately. In general, the behavior of the pressure function for $T>T_{\mathrm{c}}$ reflects the behavior of the thermal bosonic mass $m_{\phi}$. In Fig. 4 we show $m_{\phi} / T$ as a function of $T / T_{\mathrm{c}}$ as obtained from LPA as well as from the truncations AC. For asymptotically high temperatures we expect that $m_{\phi} / T=$ const. according to perturbation theory [58]. We find that this limit is not yet reached for the temperature range studied here. Moreover, we observe a difference between the results from LPA and from truncation $\mathrm{C}$. The difference between the results from truncation $\mathrm{B}$ and truncation $\mathrm{C}$, where we have also resolved the difference in the running of the wave-function renormalizations in the Euclidean time and space directions, is small for $T / T_{\mathrm{c}} \lesssim 2$. This represents an a posteriori justification for many studies where $Z_{\phi, \psi}^{\|} \equiv Z_{\phi, \psi}^{\perp}$ has been assumed, see e. g. Refs. [30, 38, 39, 59]. For a high-accuracy study of critical dynamics, however, our results suggest that higher-order derivatives may still play a role in the vicinity of the phase transition temperature. Therefore an inclusion of the full momentum dependence of the vertices is important for such a high-accuracy study. At vanishing temperature, the momentum dependence has been fully resolved in a study of critical exponents of $O(N)$ models which has led to accurate predictions [60].

Let us now discuss the results obtained from our large$N_{c}$ approximation. In Fig. 5 we compare our results for 


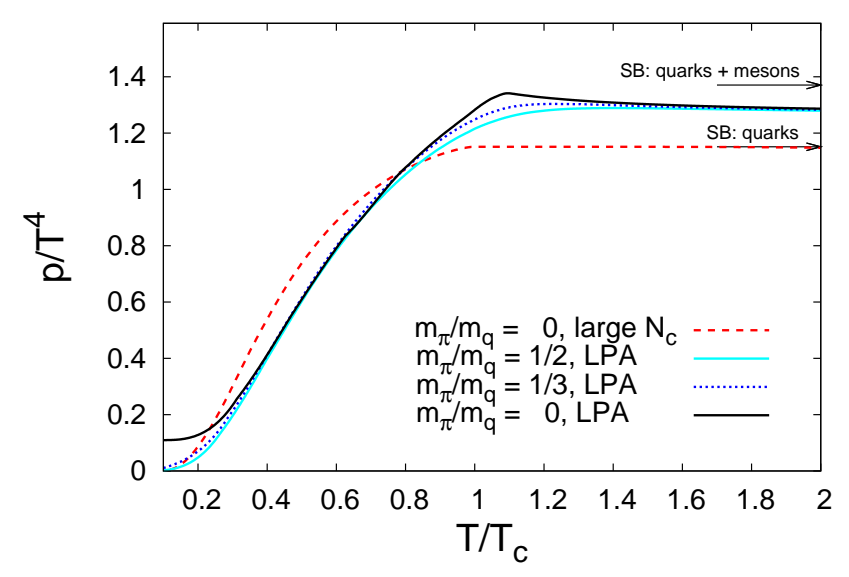

Figure 5: (Color online) Pressure as a function of $T / T_{\mathrm{c}}$ for a large- $N_{c}$ approximation of our RG flow equations and for LPA for various ratios $m_{\pi} / m_{q}=0, \frac{1}{3}, \frac{1}{2}$.

the pressure from a large- $N_{c}$ approximation in the chiral limit with the results from LPA for $m_{\pi} / m_{q}=0,1 / 3,1 / 2$. In particular $m_{\pi} / m_{q}=1 / 3$ resembles the situation in QCD with two quark flavors, where we have pions with a mass of roughly $130 \mathrm{MeV}$ and constituent quarks with a mass of roughly $330 \mathrm{MeV}$. In large $N_{c}$ and LPA with $m_{\pi} / m_{q}>0$, we find that the pressure goes to zero for small temperatures since bosonic fluctuations are suppressed. With increasing temperature the pressure increases. In contrast to the results from LPA, the large$N_{c}$ pressure function reaches its maximal value already at $T=T_{\mathrm{c}}$. Thus, the large- $N_{c}$ results do not exhibit a maximum for $T>T_{\mathrm{c}}$. Comparing them with the results from LPA with a finite mass for the (pseudo-) Goldstone boson, we find that the scalar fields still contribute significantly to the pressure of the system for $T / T_{\mathrm{c}} \lesssim 2$, even for comparatively large mass ratios $m_{\pi} / m_{q} \sim 1 / 2$. This is due to the fact that the thermal contributions to the masses of the scalar fields dominate for $T>T_{\max }$. Therefore we conclude that in our studies the pressure below $T_{c}$ is mostly affected by the the presence of a finite quark mass. However, the maximum of the pressure is still clearly visible even though it is washed out compared to the chiral limit.

Finally we comment on a possible dependence of our results on the UV cutoff $\Lambda$. We illustrate such a dependence using our results for the large- $N_{c}$ approximation as an example, but we have checked for the other truncations as well that our results are not spoiled by the presence of the UV cutoff. In Fig. 6, we show the large- $N_{c}$ result for the pressure as a function of temperature for $f_{\pi} / \Lambda=0.060,0.030,0.020,0.015$. In the studied temperature range we find that the results for the pressure are strongly affected by the presence of the UV cutoff for $f_{\pi} / \Lambda \gtrsim 0.020$. Note that we have chosen a $3 d$ regulator function which allows us to perform the Matsubara sum analytically. Nonetheless the UV cutoff still enters into the thermal distribution functions in the flow equation (7) due to their dependence on $T / k$. In order to

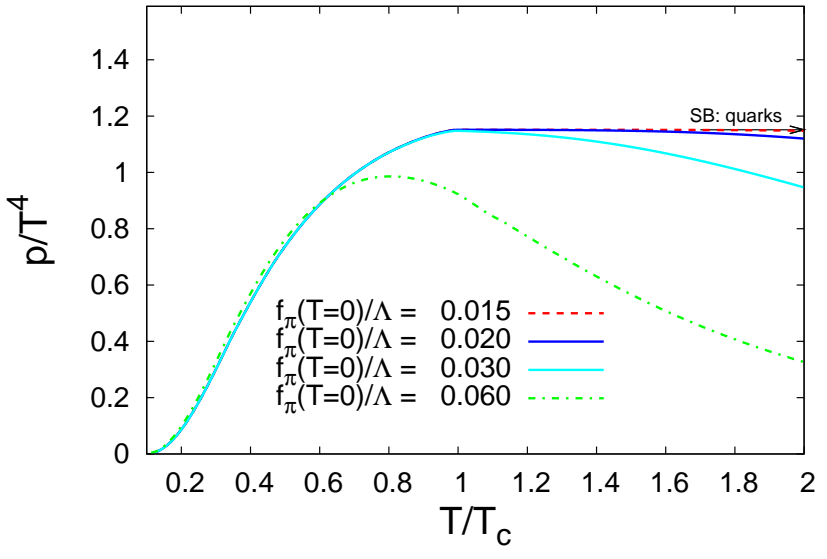

Figure 6: (Color online) Pressure as a function of $T / T_{\mathrm{c}}$ for various values of the UV cutoff $\Lambda$ as obtained from the RG flow equations in a large- $N_{c}$ approximation. The parameters of the model for the various values of $\Lambda$ have been adjusted such that the IR physics at $\mathrm{T}=0$ remain unchanged.

ensure that our results are not affected by the cutoff, we have chosen $f_{\pi} / \Lambda=0.015$ in the present work. Note that in the large- $N_{c}$ approximation the cutoff dependence of the thermodynamic observables, e. g. the pressure, can in principle be fixed by simply adding the missing contribution (at least in the chiral limit), see e. g. Ref. [16]:

$$
\frac{p_{\Lambda}}{T^{4}}=\frac{4 N_{c} N_{f}}{6 \pi^{2}} \int_{\Lambda}^{\infty} d x \frac{x^{3}}{\mathrm{e}^{x}+1} .
$$

As soon as we allow for momentum dependent vertices and/or finite quark masses such a simple prescription can no longer be found.

\section{CONCLUSIONS AND OUTLOOK}

We have discussed the truncation dependence of the thermodynamics of a simple model consisting of strongly interacting relativistic bosons and chiral fermions. Our results indicate that the derivative expansion is a good and valid approximation for a study of the thermal properties of Yukawa-type theories, such as the pressure or the behavior of the order parameter below the critical temperature. We find that the pressure exhibits a maximum above the critical temperature when we include bosonic fluctuations. The origin of the maximum is related to the fact that the thermal masses of the scalar fields are still small in a small window above the critical temperature and therefore contribute significantly to the equation of state. For very high temperatures, the pressure then approaches the Stefan-Boltzmann limit of a gas of free massless fermions from above. The convergence to the Stefan-Boltzmann limit is faster the better the momentum dependence of the vertices is resolved.

On the other hand, we have also shown that the critical temperature shows a stronger dependence on a variation of the truncation. In order to clarify whether our results 
for $T_{\mathrm{c}}$ can be considered as having almost converged, we eventually need to fully resolve the momentum dependence of the propagators and vertices as it has been successfully done in Refs. 606, 61, 62, 63, 64, 65, 66, 67] at vanishing temperature. This is deferred to future work.

Whether the present findings, in particular the behavior of the pressure above $T_{\mathrm{c}}$, are visible in the thermodynamic pressure of QCD can of course not be answered by the model used in this study. First of all, the model is not confining and therefore the pressure below $T_{\mathrm{c}}$ is overestimated. Within a functional RG approach, this can be resolved by combining the findings of recent functional RG studies of QCD including gauge degrees of freedom [28, 30, 68, 69]. This would allow us to compute the pressure from first principles in the sense that it would rely on only a single input parameter, namely the value of the strong coupling at the initial RG scale. Above the critical temperature, the pressure is mainly dominated by the presence of gluons. Still, the contribution from the mesons in the vicinity of the phase transition can be estimated from Eq. (23) to yield a $10 \%$ correction in QCD with two massless quark flavors. Therefore it might be possible to observe this effect in future lattice or functional RG studies of the thermodynamic pressure.

\section{Acknowledgments}

The author is very grateful to Holger Gies, Bertram Klein and Jan Martin Pawlowski for enlightening discussions and useful comments on the manuscript. This work was partly supported by the Natural Sciences and Engineering Research Council (NSERC) and by the National Research Council of Canada.

\section{Appendix A: DECOMPOSITION OF MOMENTUM TENSORS AT FINITE TEMPERATURE}

Throughout this paper we encounter integrals of the following type

$$
\mathcal{I}(T)=T \sum_{n=-\infty}^{\infty} \int \frac{d^{d-1}}{(2 \pi)^{3}} p_{\mu} p_{\nu} f\left(p_{0}^{2}, \vec{p}^{2}\right),
$$

where $T$ denotes the temperature, $p_{0}$ is a discrete variable depending on an integer $n$ and the function $f(.,$.$) sim-$ ply represents a placeholder for a general function which is symmetric under $p_{\mu} \rightarrow-p_{\mu}$. Due to symmetry, the tensor structure can be simplified as follows:

$$
p_{\mu} p_{\nu} \rightarrow p_{0}^{2} P_{\mu \nu}^{\|}+\frac{\vec{p}^{2}}{d-1} P_{\mu \nu}^{\perp}
$$

where $n_{\mu}=(1, \overrightarrow{0})$ denotes the heat-bath velocity and the transversal $(\perp)$ and the longitudinal $(\|)$ projectors are defined as follows:

$$
P_{\mu \nu}^{\|}=n_{\mu} n_{\nu} \quad \text { and } \quad P_{\mu \nu}^{\perp}=\delta_{\mu \nu}-P_{\mu \nu}^{\|} .
$$

\section{Appendix B: THRESHOLD FUNCTIONS}

The regulator dependence of the flow equations is controlled by (dimensionless) threshold functions which arise from Feynman graphs, incorporating fermionic and/or bosonic fields. Let us first introduce the so-called dimensionless regulator-shape function $r_{\phi}(x)$ and $r_{\psi}(x)$ for bosonic and fermionic fields. These functions are implicitly defined by the regulator function $R_{i}$ as follows:

$$
R_{\phi}\left(p_{0},\left\{p_{i}\right\}\right)=Z_{\phi}^{\perp}\left(p_{0},\left\{p_{i}\right\}\right) \vec{p}^{2} r_{B}\left(\vec{p}^{2} / k^{2}\right)
$$

for the bosonic fields and

$$
R_{\psi}\left(p_{0},\left\{p_{i}\right\}\right)=Z_{\psi, k}^{\perp}\left(p_{0},\left\{p_{i}\right\}\right) \not \vec{p} r_{\psi}\left(\vec{p}^{2} / k^{2}\right)
$$

for the fermionic fields.

In this work, we employ a $3 d$ optimized regulator-shape function [16, 44, 45]:

$$
r_{\phi}(x)=\left(\frac{1}{x}-1\right) \Theta(1-x)
$$

and

$$
r_{\psi}(x)=\left(\frac{1}{\sqrt{x}}-1\right) \Theta(1-x) .
$$

In the following, we use these regulator shape functions whenever we evaluate the integrals and sums in our general definitions of the threshold functions. Those threshold functions which are not defined below can be found in Ref. 30].

In order to define the threshold functions, it is convenient to define dimensionless propagators for the scalar fields $(\phi)$ and the fermions $(\psi)$, respectively:

$$
\begin{aligned}
\tilde{G}_{\phi}\left(x_{0}^{\phi}, \omega\right) & =\frac{1}{\hat{z}_{\phi} x_{0}^{\phi}+x\left(1+r_{\phi}\right)+\omega} \\
\text { and } \quad \tilde{G}_{\psi}\left(x_{0}^{\psi}, \omega\right) & =\frac{1}{\hat{z}_{\psi}^{2} x_{0}^{\psi}+x\left(1+r_{\psi}\right)^{2}+\omega},
\end{aligned}
$$

where $x_{0}^{\phi}=2 \pi n \tilde{t}$ and $x_{0}^{\psi}=2 \pi(n+1 / 2) \tilde{t}$. Note that the Matsubara modes are dressed with the ratio of the wavefunction renormalizations longitudinal and transversal to the heat-bath, $\hat{z}_{\phi}=Z_{\phi}^{\|} / Z_{\phi}^{\perp}$ and $\hat{z}_{\psi}=Z_{\psi}^{\|} / Z_{\psi}^{\perp}$. Moreover it is convenient to define the dimensionless propagators as obtained from the application of the optimized regulatorshape function defined in Eqs. (B3) and (B4):

$$
\begin{aligned}
\mathcal{G}_{\phi}\left(x_{0}^{\phi}, \omega\right) & =\frac{1}{1+\hat{z}_{\phi} x_{0}^{\phi}+\omega} \\
\text { and } \quad \mathcal{G}_{\psi}\left(x_{0}^{\psi}, \omega\right) & =\frac{1}{1+\hat{z}_{\psi}^{2} x_{0}^{\psi}+\omega} .
\end{aligned}
$$


For the wave-function renormalization of the scalar fields, we find:

$$
\begin{gathered}
\mathcal{M}_{4, \perp}^{(F)}\left(\tilde{t}, \omega ; \eta_{\psi}\right) \\
=(d-1) \tilde{t} \sum_{n=-\infty}^{\infty} \int_{0}^{\infty} d x x^{\frac{d-3}{2}} \tilde{\partial}_{t}\left\{x\left(1+r_{\psi}\right) \tilde{G}_{\psi}\left(x_{0}^{\psi}, \omega_{\psi}\right) \times\right. \\
\times\left[\frac{2 x}{d-1}\left(\frac{d^{2}}{d x^{2}}\left(1+r_{\psi}\right) \tilde{G}_{\psi}\left(x_{0}^{\psi}, \omega_{\psi}\right)\right)\right. \\
\left.\quad+\frac{d+1}{d-1}\left(\frac{d}{d x}\left(1+r_{\psi}\right) \tilde{G}_{\psi}\left(x_{0}^{\psi}, \omega_{\psi}\right)\right)\right] \\
+\hat{z}_{\psi}^{2} x_{0}^{\psi} \tilde{G}_{\psi}\left(x_{0}^{\psi}, \omega_{\psi}\right)\left[\frac{2 x}{d-1}\left(\frac{d^{2}}{d x^{2}} \tilde{G}_{\psi}\left(x_{0}^{\psi}, \omega_{\psi}\right)\right)\right. \\
\left.\left.+\left(\frac{d}{d x} \tilde{G}_{\psi}\left(x_{0}^{\psi}, \omega_{\psi}\right)\right)\right]\right\} . \quad \text { (B9) }
\end{gathered}
$$

Employing the optimized $3 d$ regulator function specified above, we obtain

$$
\begin{aligned}
& \mathcal{M}_{4, \perp}^{(F)}(\tilde{t}, \omega) \\
& =4 \tilde{t} \sum_{n=-\infty}^{\infty}\left\{\left(1+z_{\psi}^{2} x_{0}^{\psi}\right)\left(\mathcal{G}_{\psi}\left(x_{0}^{\psi}, \omega_{\psi}\right)\right)^{4}\right. \\
& +\frac{1}{d-3}\left(\mathcal{G}_{\psi}\left(x_{0}^{\psi}, \omega_{\psi}\right)\right)^{3}-\left(\frac{1}{2 d-6}+\frac{1}{4}\right)\left(\mathcal{G}_{\psi}\left(x_{0}^{\psi}, \omega_{\psi}\right)\right)^{2} \\
& \left.\quad-\frac{\eta_{\psi}^{\perp}}{d-3}\left(\mathcal{G}_{\psi}\left(x_{0}^{\psi}, \omega_{\psi}\right)\right)^{3}+\frac{\eta_{\psi}^{\perp}}{2 d-6}\left(\mathcal{G}_{\psi}\left(x_{0}^{\psi}, \omega_{\psi}\right)\right)^{2}\right\} .
\end{aligned}
$$

The threshold function for the corresponding longitudinal contribution reads:

$$
\begin{aligned}
& \mathcal{M}_{4, \|}^{(F)}(\tilde{t}, \omega) \\
& =\tilde{t} \sum_{n=-\infty}^{\infty} \int_{0}^{\infty} d x x^{\frac{d-3}{2}} \tilde{\partial}_{t}\left\{x\left(1+r_{\psi}\right) \tilde{G}_{\psi}\left(x_{0}^{\psi}, \omega_{\psi}\right) \times\right. \\
& \times\left[2 x_{0}^{\psi}\left(\frac{d^{2}}{\left(d x_{0}^{\psi}\right)^{2}}\left(1+r_{\psi}\right) \tilde{G}_{\psi}\left(x_{0}^{\psi}, \omega_{\psi}\right)\right)\right. \\
& +\left(\frac{d}{\left.\left.d x_{0}^{\psi}\left(1+r_{\psi}\right) \tilde{G}_{\psi}\left(x_{0}^{\psi}, \omega_{\psi}\right)\right)\right]}\right. \\
& +\hat{z}_{\psi}^{2} x_{0}^{\psi} \tilde{G}_{\psi}\left(x_{0}^{\psi}, \omega_{\psi}\right)\left[2\left(\frac{d^{2}}{\left(d x_{0}^{\psi}\right)^{2}} \tilde{G}_{\psi}\left(x_{0}^{\psi}, \omega_{\psi}\right)\right)\right. \\
& \left.\left.+3\left(\frac{d}{d x_{0}^{\psi}} \tilde{G}_{\psi}\left(x_{0}^{\psi}, \omega_{\psi}\right)\right)\right]\right\}
\end{aligned}
$$

$$
\begin{gathered}
=z_{\psi}^{2} \tilde{t} \sum_{n=-\infty}^{\infty} \frac{2}{d-1}\left(1-\frac{\eta_{\psi}^{\perp}}{d}\right)\left\{-2\left(\mathcal{G}_{\psi}\left(x_{0}^{\psi}, \omega_{\psi}\right)\right)^{3}\right. \\
+\left(26 \hat{z}_{\psi}^{2} x_{0}^{\psi}+6\right)\left(\mathcal{G}_{\psi}\left(x_{0}^{\psi}, \omega_{\psi}\right)\right)^{4} \\
\left.-32 \hat{z}_{\psi}^{2} x_{0}^{\psi}\left(1+\hat{z}_{\psi}^{2} x_{0}\right)\left(\mathcal{G}_{\psi}\left(x_{0}^{\psi}, \omega_{\psi}\right)\right)^{5}\right\}
\end{gathered}
$$

Apart from these contributions, the scalar wave-function renormalization also gets a contribution from a fermion loop which is proportional to the vacuum expectation value of the scalar field:

$$
\begin{gathered}
\mathcal{M}_{2, \|}^{(F)}\left(\tilde{t}, \omega_{\psi}\right)=\frac{1}{4} \hat{z}_{\psi}^{2} \tilde{t} \sum_{n=-\infty}^{\infty} \int_{0}^{\infty} d x x^{\frac{d-3}{2}} \tilde{\partial}_{t} \tilde{G}_{\psi}\left(x_{0}^{\psi}, \omega_{\psi}\right) \times \\
\times\left\{\left(\frac{d}{d x_{0}^{\psi}} \tilde{G}_{\psi}\left(x_{0}^{\psi}, \omega_{\psi}\right)\right)\right. \\
\left.+2 \hat{z}_{\psi}^{2} x_{0}^{\psi}\left(\frac{d^{2}}{\left(d x_{0}^{\psi}\right)^{2}} \tilde{G}_{\psi}\left(x_{0}^{\psi}, \omega_{\psi}\right)\right)\right\} \\
=\frac{1}{d-1}\left(1-\frac{\eta_{\psi}^{\perp}}{d}\right) \hat{z}_{\psi}^{2} \tilde{t} \sum_{n=-\infty}^{\infty}\left(\mathcal{G}_{\psi}\left(x_{0}^{\psi}, \omega_{\psi}\right)\right)^{4} \times \\
\times\left\{3-16 \hat{z}_{\psi}^{2} x_{0}^{\psi} \mathcal{G}_{\psi}\left(x_{0}^{\psi}, \omega_{\psi}\right)\right\} \times \quad(\mathrm{B} 12
\end{gathered}
$$

Finally, there is a contribution to the scalar wavefunction renormalization from a pion-sigma loop which is proportional to the four-boson coupling:

$$
\begin{aligned}
& \mathcal{M}_{2,2, \|}^{(B)}\left(\tilde{t}, \omega_{\pi}, \omega_{\sigma}\right) \\
& =\frac{\hat{z}_{\phi} \tilde{t}}{8} \sum_{n=-\infty}^{\infty} \int_{0}^{\infty} d x x^{\frac{d-3}{2}} \tilde{\partial}_{t} \mathcal{G}_{\phi}\left(x_{0}^{\phi}, \omega_{\phi, 1}\right)\left(\mathcal{G}_{\pi}\left(x_{0}^{\phi}, \omega_{\sigma}\right)\right)^{2} \times \\
& \quad \times\left\{8 \hat{z}_{\phi} x_{0}^{\phi} \mathcal{G}_{\phi}\left(x_{0}^{\phi}, \omega_{\sigma}\right)-2\right\}+\left(\omega_{\pi} \leftrightarrow \omega_{\sigma}\right) \\
& =\left(1-\frac{\eta_{\phi}^{\perp}}{d+1}\right) \frac{\hat{z}_{\phi} \tilde{t}}{(d-1)} \sum_{n=-\infty}^{\infty}\left\{\mathcal{G}_{\phi}\left(x_{0}^{\phi}, \omega_{\pi}\right) \times\right. \\
& \quad \times\left(\mathcal{G}_{\phi}\left(x_{0}^{\phi}, \omega_{\sigma}\right)\right)^{2}\left[2 \mathcal{G}_{\phi}\left(x_{0}^{\phi}, \omega_{\sigma}\right)+\mathcal{G}_{\phi}\left(x_{0}^{\phi}, \omega_{\pi}\right)\right] \\
& -4 \hat{z}_{\phi} x_{0}^{\phi} \mathcal{G}_{\phi}\left(x_{0}^{\phi}, \omega_{\pi}\right)\left(\mathcal{G}_{\phi}\left(x_{0}^{\phi}, \omega_{\sigma}\right)\right)^{3}\left[\mathcal{G}_{\phi}\left(x_{0}^{\phi}, \omega_{\pi}\right)\right. \\
& \left.\left.+3 \mathcal{G}_{\phi}\left(x_{0}^{\phi}, \omega_{\sigma}\right)\right]\right\}+\left(\omega_{\pi} \leftrightarrow \omega_{\sigma}\right) . \quad(\mathrm{B} 13)
\end{aligned}
$$

For the fermionic wave-function renormalization longitudinal to the heat-bath, we find

$$
\begin{gathered}
\mathcal{M}_{1,2, \|}^{(F B)}\left(\tilde{t}, \omega_{\psi}, \omega_{\phi}\right) \\
=\frac{\tilde{t}}{2} \sum_{n=-\infty}^{\infty} \int_{0}^{\infty} d x x^{\frac{d-3}{2}} \tilde{\partial}_{t} \frac{\hat{z}_{\psi} x_{0}^{\psi}}{\left(1+r_{\psi}\right)}\left\{-\tilde{G}_{\psi}\left(x_{0}^{\psi}, \omega_{\psi}\right) \times\right. \\
\left.\times\left(\frac{d}{d x_{0}^{\psi}} \tilde{G}_{\phi}\left(x_{0}^{\psi}, \omega_{\phi}\right)\right)\right\}
\end{gathered}
$$




$$
\begin{gathered}
=\frac{2}{d-1} \hat{z}_{\psi} \hat{z}_{\phi} \tilde{t} \sum_{n=-\infty}^{\infty} x_{0}^{\psi} \mathcal{G}_{\psi}\left(x_{0}^{\psi}, \omega_{\psi}\right)\left(\mathcal{G}_{\phi}\left(x_{0}^{\psi}, \omega_{\phi}\right)\right)^{2} \times \\
\times\left\{\left(1-\frac{\eta_{\psi}^{\perp}}{d}\right) \mathcal{G}_{\psi}\left(x_{0}^{\psi}, \omega_{\psi}\right)+2\left(1-\frac{\eta_{\phi}^{\perp}}{d+1}\right) \mathcal{G}_{\phi}\left(x_{0}^{\psi}, \omega_{\phi}\right)\right\} .
\end{gathered}
$$

Note that the Matsubara sums in the threshold func- tions can be performed analytically. Since the resulting expressions are rather complicated and of no interest for the discussions in the present paper, we do not show them here. However, for the numerical evaluation of the RG flows we have made use of the fact that the Matsubara sum in these functions can be performed analytically.
[1] P. Braun-Munzinger, J. Stachel, and C. Wetterich, Phys. Lett. B596, 61 (2004), nucl-th/0311005.

[2] T. Appelquist and R. D. Pisarski, Phys. Rev. D23, 2305 (1981).

[3] K. Kajantie, M. Laine, K. Rummukainen, and M. E. Shaposhnikov, Nucl. Phys. B458, 90 (1996), hep$\mathrm{ph} / 9508379$.

[4] R. D. Pisarski, Physica A158, 246 (1989).

[5] R. D. Pisarski, Phys. Rev. Lett. 63, 1129 (1989).

[6] J. Frenkel and J. C. Taylor, Nucl. Phys. B334, 199 (1990).

[7] E. Braaten and R. D. Pisarski, Nucl. Phys. B339, 310 (1990).

[8] M. H. Thoma (2000), hep-ph/0010164.

[9] J.-P. Blaizot and E. Iancu, Phys. Rept. 359, 355 (2002), hep-ph/0101103.

[10] J.-P. Blaizot, E. Iancu, and A. Rebhan (2003), hep$\mathrm{ph} / 0303185$.

[11] F. Di Renzo, M. Laine, V. Miccio, Y. Schroder, and C. Torrero, JHEP 07, 026 (2006), hep-ph/0605042.

[12] Y. Nambu and G. Jona-Lasinio, Phys. Rev. 122, 345 (1961).

[13] P. N. Meisinger and M. C. Ogilvie, Phys. Lett. B379, 163 (1996), hep-lat/9512011.

[14] R. D. Pisarski, Phys. Rev. D62, 111501 (2000), hep$\mathrm{ph} / 0006205$.

[15] K. Fukushima, Phys. Lett. B591, 277 (2004), hep$\mathrm{ph} / 0310121$.

[16] J. Braun, K. Schwenzer, and H.-J. Pirner, Phys. Rev. D70, 085016 (2004), hep-ph/0312277.

[17] C. Ratti, M. A. Thaler, and W. Weise, Phys. Rev. D73, 014019 (2006), hep-ph/0506234.

[18] E. Megias, E. Ruiz Arriola, and L. L. Salcedo, Phys. Rev. D74, 065005 (2006), hep-ph/0412308.

[19] C. Sasaki, B. Friman, and K. Redlich, Phys. Rev. D75, 074013 (2007), hep-ph/0611147.

[20] B.-J. Schaefer, J. M. Pawlowski, and J. Wambach, Phys. Rev. D76, 074023 (2007), arXiv:0704.3234 [hep-ph].

[21] C. Wetterich, Phys. Lett. B301, 90 (1993).

[22] G. 't Hooft, Phys. Rev. D14, 3432 (1976).

[23] M. A. Shifman, A. I. Vainshtein, and V. I. Zakharov, Nucl. Phys. B163, 46 (1980).

[24] E. V. Shuryak, Nucl. Phys. B203, 93 (1982).

[25] T. Schafer and E. V. Shuryak, Rev. Mod. Phys. 70, 323 (1998), hep-ph/9610451.

[26] J. Meyer, K. Schwenzer, H.-J. Pirner, and A. Deandrea, Phys. Lett. B526, 79 (2002), hep-ph/0110279.

[27] M. Buballa, Phys. Rept. 407, 205 (2005), hep$\mathrm{ph} / 0402234$.

[28] H. Gies and C. Wetterich, Phys. Rev. D69, 025001
(2004), hep-th/0209183.

[29] J. M. Pawlowski, Annals Phys. 322, 2831 (2007), hepth/0512261.

[30] J. Braun (2008), 0810.1727.

[31] H. Gies and C. Wetterich, Phys. Rev. D65, 065001 (2002), hep-th/0107221.

[32] S. Floerchinger and C. Wetterich (2009), 0905.0915.

[33] B.-J. Schaefer and H.-J. Pirner, Nucl. Phys. A660, 439 (1999), nucl-th/9903003.

[34] B.-J. Schaefer and J. Wambach, Nucl. Phys. A757, 479 (2005), nucl-th/0403039.

[35] J. Braun, B. Klein, H. J. Pirner, and A. H. Rezaeian, Phys. Rev. D73, 074010 (2006), hep-ph/0512274.

[36] G. Von Gersdorff and C. Wetterich, Phys. Rev. B64, 054513 (2001), hep-th/0008114.

[37] N. Tetradis and C. Wetterich, Nucl. Phys. B422, 541 (1994), hep-ph/9308214.

[38] J. Berges, D. U. Jungnickel, and C. Wetterich, Phys. Rev. D59, 034010 (1999), hep-ph/9705474.

[39] J. Berges, N. Tetradis, and C. Wetterich, Phys. Rept. 363, 223 (2002), hep-ph/0005122.

[40] J. Braun and B. Klein, Phys. Rev. D77, 096008 (2008), 0712.3574 .

[41] J. Braun and B. Klein (2008), 0810.0857.

[42] E. Nakano, B. J. Schaefer, B. Stokic, B. Friman, and K. Redlich (2009), 0907.1344.

[43] G. Papp, B. J. Schaefer, H. J. Pirner, and J. Wambach, Phys. Rev. D61, 096002 (2000), hep-ph/9909246.

[44] D. F. Litim and J. M. Pawlowski, JHEP 11, 026 (2006), hep-th/0609122.

[45] J.-P. Blaizot, A. Ipp, R. Mendez-Galain, and N. Wschebor, Nucl. Phys. A784, 376 (2007), hep-ph/0610004.

[46] D. F. Litim and J. M. Pawlowski, Phys. Lett. B516, 197 (2001), hep-th/0107020.

[47] D. F. Litim, Phys. Lett. B486, 92 (2000), hepth/0005245.

[48] D. F. Litim, Phys. Rev. D64, 105007 (2001), hepth/0103195.

[49] D. F. Litim and J. M. Pawlowski (1998), hep-th/9901063.

[50] J. Polonyi, Central Eur. J. Phys. 1, 1 (2003), hepth/0110026.

[51] H. Gies (2006), hep-ph/0611146.

[52] D. U. Jungnickel and C. Wetterich, Phys. Rev. D53, 5142 (1996), hep-ph/9505267.

[53] H. Gies, S. Rechenberger, and M. M. Scherer (2009), 0907.0327.

[54] D. F. Litim, Nucl. Phys. B631, 128 (2002), hepth/0203006.

[55] J. Braun, B. Klein, and H. J. Pirner, Phys. Rev. D71, 014032 (2005), hep-ph/0408116. 
[56] J. Braun and H. Gies, Phys. Lett. B645, 53 (2007), hep$\mathrm{ph} / 0512085$.

[57] J. Braun and H. Gies, JHEP 06, 024 (2006), hep$\mathrm{ph} / 0602226$.

[58] L. Dolan and R. Jackiw, Phys. Rev. D9, 3320 (1974).

[59] O. Bohr, B. J. Schaefer, and J. Wambach, Int. J. Mod. Phys. A16, 3823 (2001), hep-ph/0007098.

[60] F. Benitez et al. (2009), 0901.0128.

[61] U. Ellwanger, M. Hirsch, and A. Weber, Z. Phys. C69, 687 (1996), hep-th/9506019.

[62] J. M. Pawlowski, D. F. Litim, S. Nedelko, and L. von Smekal, Phys. Rev. Lett. 93, 152002 (2004), hepth/0312324.

[63] C. S. Fischer and H. Gies, JHEP 10, 048 (2004), hep- $\mathrm{ph} / 0408089$.

[64] J. P. Blaizot, R. Mendez Galain, and N. Wschebor, Phys. Lett. B632, 571 (2006), hep-th/0503103.

[65] J.-P. Blaizot, R. Mendez-Galain, and N. Wschebor, Phys. Rev. E74, 051116 (2006), hep-th/0512317.

[66] J.-P. Blaizot, R. Mendez-Galain, and N. Wschebor, Phys. Rev. E74, 051117 (2006), hep-th/0603163.

[67] S. Diehl, H. C. Krahl, and M. Scherer, Phys. Rev. C78, 034001 (2008), 0712.2846.

[68] J. Braun, H. Gies, and J. M. Pawlowski (2007), arXiv:0708.2413 [hep-th].

[69] J. Braun, L. M. Haas, F. Marhauser, and J. M. Pawlowski (2009), 0908.0008. 